\title{
Antagonismo das Associações de Clodinafop-Propargyl com Metsulfuron-Methyl e 2,4-D no Controle de Azevém (Lolium multiflorum) ${ }^{1}$
}

\author{
Antagonistic Action of Clodinafop-Propargyl Associated with Metsulfuron-Methyl and 2,4-D in \\ the Control of Italian Ryegrass (Lolium multiflorum)
}

\author{
TREZZI, M.M. ${ }^{2}$, MATTEI, D. ${ }^{3}$, VIDAL, R.A. ${ }^{4}$, KRUSE, N.D. ${ }^{5}$, GUSTMAN, M.S. ${ }^{6}$, VIOLA, R. ${ }^{6}$, \\ MACHADO, A. ${ }^{6}$ e SILVA, H.L. ${ }^{3}$
}

\begin{abstract}
RESUMO - Em geral, a eficiência de herbicidas inibidores da ACCase é reduzida quando da aplicação em conjunto com herbicidas latifolicidas. Dois experimentos - em casa de vegetação e campo - objetivaram determinar a existência de antagonismo nas associações de clodinafop-propargyl a metsulfuron-methyl ou 2,4-D, para controle de azevém anual. Em casa de vegetação, o delineamento foi o completamente casualizado, com 21 tratamentos compostos pela aplicação de clodinafop $\left(0,3,6,12,24,48\right.$ e $\left.96 \mathrm{~g} \mathrm{ha}^{-1}\right)$ isolado ou em mistura com metsulfuron-methyl (2 e $4 \mathrm{~g} \mathrm{ha}^{-1}$ ) ou 2,4-D (470 $\left.\mathrm{g} \mathrm{ha}^{-1}\right)$. Em campo, o delineamento foi de blocos casualizados, com 12 tratamentos, arranjados em fatorial $6 \times 2$, composto pelos niveis de clodinafop-propargyl $\left(0,3,6,12,24,48\right.$ e $96 \mathrm{~g}$ ha $\left.{ }^{1}\right)$ isolado ou em mistura com metsulfuron-methyl ( $\left.2 \mathrm{~g} \mathrm{ha}^{-1}\right)$. A avaliação visual de controle, em casa de vegetação, revelou $\mathrm{I}_{50}$ das misturas de clodinafop-propargyl + metsulfuron-methyl (2 e $4 \mathrm{~g} \mathrm{ha}^{-1}$ ) ou clodinafoppropargyl + 2,4-D (470 $\left.\mathrm{g} \mathrm{ha}^{-1}\right)$, respectivamente, 33, 84 e 151\% superiores ao de clodinafoppropargyl isolado. Já para matéria verde de azevém, o $\mathrm{I}_{50}$ das misturas supracitadas foi, respectivamente, 119, 244 e $72 \%$ superior ao de clodinafop-propargyl isolado. Em campo, ocorreu redução da matéria verde de azevém com a elevação dos níveis de clodinafop-propargyl isolado, mas não houve variação da matéria verde com a elevação dos níveis de clodinafoppropargyl, em associação a metsulfuron-methyl. Os resultados evidenciam a existência de antagonismo entre clodinafop-propargyl e os herbicidas metsulfuron-methyl e 2,4-D. São feitas considerações sobre as possiveis vantagens da aplicação dos herbicidas em mais de uma operação de controle, em comparação à associação entre graminicidas e latifolicidas.
\end{abstract}

Palavras-chave: associação de herbicidas, inibidores da ACCase, inibidores da ALS, auxinas sintéticas.

\begin{abstract}
The efficacy of ACCase inhibitors is usually reduced when tank-mixed with broad leaf herbicides. A greenhouse and a field experiments were carried out to determine the existence of antagonistic effects of clodinafop-propargyl associated with metsulfuron methyl or 2,4-D to control Italian ryegrass (Lolium multiflorum). In the greenhouse experiment, 21 treatments were tested, composed by clodynafop $\left(0,3,6,12,24,48\right.$ and $\left.96 \mathrm{~g} \mathrm{ha}^{1}\right)$ alone or tank-mixed with metsulfuron (2 and $\left.4 \mathrm{~g} \mathrm{ha}^{-1}\right)$ or 2,4-D (470 $\left.\mathrm{g} \mathrm{ha}^{-1}\right)$. In the field experiment, twelve treatments were set up in a factorial $6 \times 2$, composed by clodinafop $\left(0,3,6,12,24,48\right.$ and $\left.96 \mathrm{~g} \mathrm{ha}^{-1}\right)$ alone or tank-

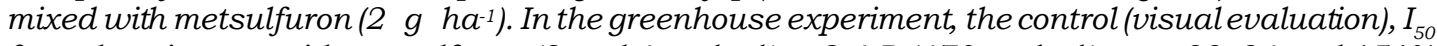

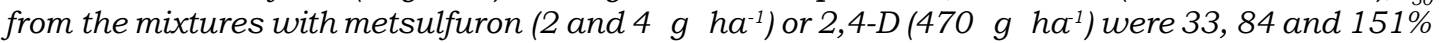
higher than clodinafop applied alone. The $I_{50}$ from the rye live matter from the mixtures cited above
\end{abstract}

Recebido para publicação em 13.4.2007 e na forma revisada em 29.11.2007.

2 Eng-Agr - - , Dr., Professor do Curso de Agronomia da Universidade Tecnológica Federal do Paraná (UTFPR), Campus Pato Branco, Via do Conhecimento, km 01, Caixa Postal 571, Pato Branco-PR, <trezzi@utfpr.edu.br>; ${ }^{3}$ Eng-Agr ${ }^{\circ}$, mestrando do Programa de Pós-Graduação em Agronomia da UTFPR, Campus Pato Branco; ${ }^{4}$ Engo-Agro , Ph. D., Professor da Faculdade de Agronomia da Universidade Federal do Rio Grande do Sul, Porto Alegre-RS; ${ }^{5}$ Engo-Agr ${ }^{\circ}$, Dr., Professor do Centro de Ciências Rurais da Universidade Federal de Santa Maria, Santa Maria-RS; ${ }^{6}$ Acadêmico do curso de Agronomia da UTFPR, Campus Pato Branco.

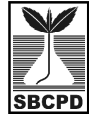

Planta Daninha, Viçosa-MG v. 25, n. 4, p. 839-847, 2007 
were, respectively, 119, 244 and 72\% higher than clodinafop applied alone. In the field experiment, rye live matter reduction was observed with increase of the levels of clodinafop applied alone, but rye live matter did not decrease, changing the levels of clodinafop tank-mixed with metsulfuron. The results reveal the antagonistic effect of tank-mixtures of clodinafop and metsulfuron or 2,4-D. We did make some considerations on the advantages of applying herbicides in more than one control operation, in comparison to graminicide and latifolicide mixtures.

Keywords: herbicide association, ACCase inhibitors, ALS inhibitors, synthetic auxines.

\section{INTRODUÇÃO}

O azevém anual (Lolium multiflorum) é uma espécie amplamente utilizada em sistemas de integração lavoura-pecuária na região Sul do Brasil. Essa espécie apresenta ressemeadura natural, o que reduz os custos e facilita sua implantação após seu primeiro cultivo em uma área. No entanto, as plantas de azevém que germinam após a operação de dessecação realizada antes da semeadura do trigo podem trazer problemas, dependendo de fatores como a densidade de plantas por área e do intervalo entre a dessecação e a semeadura da cultura. $O$ azevém compete com plantas de trigo durante as fases de crescimento vegetativo e reprodutivo e causa problemas na colheita, por se manter vegetando neste período. Densidades de azevém anuais de 29 a 118 plantas $\mathrm{m}^{-2}$ podem reduzir o rendimento de trigo em 7 a $50 \%$ (Appleby et al., 1976). A competição causada por azevém reduziu a produtividade de trigo em $4,2 \%$ para cada 10 plantas $\mathrm{m}^{-2}$ de azevém (Liebel \& Worsham, 1987). No entanto, a capacidade competitiva do trigo com azevém é bastante dependente do porte dos cultivares, como demonstrado por Fleck (1980). Esse autor observou decréscimo linear do rendimento de grãos com o aumento da população de azevém, para o cultivar E 7414 , de porte mais baixo, enquanto para PAT-7219, de porte mais alto, não houve redução do rendimento de grãos com a elevação da população.

Além disso, a contaminação de lavouras com azevém anual inviabiliza a produção de sementes de trigo. A legislação federal estabelece que deve haver a eliminação completa de plantas de azevém em campos de sementes de trigo (Brasil, 2005). Por esses motivos, torna-se necessário o desenvolvimento de um sistema capaz de eliminar ou reduzir os níveis dessa espécie invasora do trigo; do contrário, torna-se dificil aumentar a produtividade das áreas e manter ou ampliar as áreas de produção de sementes.

Os inibidores da ACCase dividem-se nos grupos químicos ariloxifenoxipropionatos e cicloexanodionas. Esses herbicidas inibem a formação de lipídios, os quais compõem de 5 a $10 \%$ do peso seco do vegetal e são importantes constituintes estruturais deste (Vidal \& Merotto Jr., 2001 ). Eles inibem reversivelmente a ACCase, impedindo a ligação dessa enzima com o $\mathrm{CO}_{2}$, que posteriormente é transferido para o grupamento acetilCoa - reação necessária à síntese de lipídios pela planta. São utilizados no controle de plantas daninhas na agricultura desde a década de 1960. Esses produtos são usados principalmente em culturas dicotiledôneas, uma vez que esses herbicidas controlam apenas espécies daninhas gramineas. Entretanto, inibidores de ACCase, como diclofop-methyl e clodinafop-propargyl, apresentam seletividade para culturas monocotiledôneas como o trigo (Vidal \& Merotto Jr., 2001; Vencill, 2002).

Até 2004, diclofop-methyl era o único inibidor da ACCase pós-emergente utilizado para controlar azevém na cultura do trigo no Brasil. A partir desse ano, foi obtido o registro emergencial de clodinafop-propargyl, inibidor da ACCase para uso em trigo. Em 2006, foi obtido o registro federal do produto comercial no Brasil. Clodinafop-propargyl é registrado também em outros países, para controle de gramineas invasoras na cultura do trigo, sem causar grande toxicidade a essa cultura (Nuòes \& Sanchez, 1998; Bailey \& Wilson, 2003; Hoskins et al., 2005). Em 2005, também passou a ser comercializado no Brasil o herbicida iodosulfuron-methyl, inibidor da ALS que também controla azevém anual em pós- 
emergência em trigo. Clodinafop-propargyl controla melhor as aveias ( $A$. sativa e $A$. strigosa) do que azevém, enquanto iodosulfuron-methyl controla melhor azevém do que aveia.

A seletividade dos inibidores da ACCase ocorre através da rápida metabolização ou por insensibilidade da enzima. Em gramineas tolerantes a esses herbicidas, como o trigo em relação ao diclofop-methyl, a tolerância se dá pela rápida metabolização. Já em dicotiledôneas essa tolerância se dá pela insensibilidade da ACCase (Vidal \& Merotto Jr., 2001). A seletividade de trigo a clodinafop-propargyl pode ser ampliada com a mistura do protetor (safener) cloquintocet-mexyl, que protege eficientemente o trigo e parcialmente a cevada, mas não possui efeitos sobre a aveia e o azevém (Vencill, 2002). A seletividade de vários niveis de clodinafop-propargyl e de um protetor a variedades de trigo cultivadas no Chile foi avaliada por Nuñes \& Sanchez (1998). Mesmo em níveis quatro vezes maiores que o recomendado, os efeitos fitotóxicos ao trigo foram pequenos e desapareceram durante o desenvolvimento da cultura.

Como clodinafop-propargyl não controla espécies latifoliadas, deve ser empregado em programas de controle que incluam herbicidas latifolicidas. As associações de graminicidas com latifolicidas racionalizam o tempo, o uso das máquinas na propriedade e o trabalho do aplicador. Um dos problemas enfrentados, nesses casos, é o antagonismo decorrente da associação, bem documentado para várias espécies cultivadas (Olson \& Nalejawa, 1981; Deschamps et al., 1990; Holshouser \& Coble, 1990; Vidrine et al., 1995; Brommer et al., 2000; Burke et al., 2002; Scherder et al., 2005).

A interação resultante da associação de clodinafop-propargyl com metsulfuron-methyl e 2,4-D - herbicidas amplamente utilizados para controlar espécies latifoliadas em trigo ainda é pouco estudada. Nos Estados Unidos, Minton et al. (1989) demonstraram que a associação de clodinafop-propargyl com herbicidas inibidores da ALS comprometeu o controle de Echinochloa crus-galli em taxas que variaram entre 5 e 45\%. Na região Sudoeste do Paraná, a associação de clodinafop-propargyl (36 $\mathrm{g} \mathrm{ha}^{-1}$ ) com metsulfuron-methyl $\left(4 \mathrm{~g} \mathrm{ha}^{-1}\right)$ reduziu o controle de azevém em $28 \%$, em relação ao clodinafop isolado (Mattei et al., 2004). Na região dos Campos Gerais do Paraná, Kunz et al. (2004) verificaram leve antagonismo entre clodinafop-propargyl e metsulfuron-methyl em aplicação seqüencial para controle de Avena sativa, efeito não observado para $A$. strigosa. Os autores relatam ainda que o herbicida 2,4$\mathrm{D}$ apresentou forte antagonismo com clodinafop-propargyl para as duas espécies avaliadas. Como clodinafop-propargyl apresenta maior eficiência sobre aveias do que sobre azevém, poder-se-ia especular que o azevém apresenta maiores problemas de antagonismo do que as espécies de aveia.

O antagonismo pode ocorrer em função da redução da absorção (Culpepper et al., 1999; Brommer et al., 2000) ou da absorção e translocação dos graminicidas pelos latifolicidas (Holshouser \& Coble, 1990). Outra possibilidade seria a redução de atividades metabólicas, como da divisão celular e do fornecimento de lipídios para formação da membrana, o que comprometeria a atividade dos inibidores de ACCase (Minton et al., 1989).

Efeitos antagônicos ocorrem principalmente quando o graminicida é aplicado juntamente ou após os herbicidas latifolicidas. Entretanto, o efeito resultante pode ser maximizado ou minimizado conforme o intervalo entre as aplicações dos herbicidas adotado no sistema (Minton et al., 1989; Culpepper et al., 1998; Scherder et al., 2005). Nas aplicações de clodinafop-propargyl, os intervalos de 12 dias antes ou após a aplicação de metsulfuron-methyl foram suficientes para evitar a redução na eficiência de clodinafop-propargyl, possibilitando, dessa forma, controle de azevém acima de $80 \%$ (Mattei et al., 2004).

Existem vários métodos para calcular sinergismo ou antagonismo entre herbicidas. O método de Colby (1967) é uma metodologia clássica, válida somente para casos em que os componentes da associação exibem ação não-similar. Outra fórmula de cálculo inclui o uso de modelos de regressão não-linear para ajuste dos resultados obtidos, principalmente as curvas de resposta do tipo log-logística (Kruse et al., 2006). Este trabalho objetivou verificar a existência de antagonismo entre clodinafoppropargyl e metsulfuron-methyl ou 2,4-D no controle de azevém anual. 


\section{MATERIAL E MÉTODOS}

O primeiro experimento foi realizado em vasos com capacidade para $1 \mathrm{~kg}$ de solo, em casa de vegetação, no Curso de Agronomia da Universidade Tecnológica Federal do Paraná (UTFPR), em Pato Branco (PR). O delineamento experimental foi completamente casualizado, com 28 tratamentos representados pelo uso de clodinafop-propargyl $(0,3,6,12,24,48$ e $\left.96 \mathrm{~g} \mathrm{ha}^{-1}\right)$ isolado ou em mistura com metsulfuron-methyl (2 e $4 \mathrm{~g} \mathrm{ha}^{-1}$ ) ou 2,4-D (469 $\left.\mathrm{g} \mathrm{ha}^{-1}\right)$, para caracterização das curvas de dose-resposta. Niveis entre 40 e $50 \mathrm{~g} \mathrm{ha}^{-1}$ de clodinafoppropargyl correspondem à faixa recomendada pelo fabricante para controle de azevém. Foi adicionado o óleo mineral Nimbus ${ }^{\circledR}$ a $0,2 \%$ para metsulfuron-methyl e $0,5 \%$ para clodinafop-propargyl e associações com outros herbicidas. O solo usado foi coletado na Área Experimental da UTFPR, peneirado e depositado nos vasos. Sementes de azevém (Lolium multiflorum) foram semeadas, obtendo-se em torno de 300 plantas $\mathrm{m}^{-2}$. Quando as plantas de azevém estavam com aproximadamente cinco folhas completamente expandidas, foram aplicados os tratamentos herbicidas. Na aplicação dos herbicidas utilizou-se pulverizador pressurizado a $\mathrm{CO}_{2}$, munido de dois bicos tipo leque 11003, utilizando-se volume de calda equivalente a $250 \mathrm{~L} \mathrm{ha}^{-1}$. A aspersão foi efetuada em ausência de vento, com temperatura média de $25^{\circ} \mathrm{C}$ e $55 \%$ de umidade relativa do ar. As plantas de azevém foram mantidas sob boa disponibilidade hídrica durante todo o periodo experimental, através de irrigação por aspersão realizada diariamente. Aos 14 dias após aplicação dos herbicidas (DAA), procedeuse à avaliação dos sintomas dos herbicidas nas plantas através de escala de 0 a $100 \%$, em que 0 representou ausência de sintomas e 100\% a morte das plantas. Aos 28 DAA, efetuou-se o corte das plantas na altura do solo e determinou-se a matéria verde.

No segundo experimento, conduzido em campo, simulou-se área de trigo infestada com azevém. Cada parcela continha 10 linhas de $4 \mathrm{~m}$ de comprimento, espaçadas de $0,2 \mathrm{~m}$. A área útil foi de $4 \mathrm{~m}^{2}$. As duas espécies (trigo e azevém) foram implantadas no mesmo dia. $\mathrm{O}$ azevém foi semeado a lanço, manualmente, ao que se seguiu a incorporação das sementes por meio de gradagem niveladora. O trigo, cultivar CD-205, foi semeado em linhas. As densidades médias obtidas foram de 400 e 50 plantas $\mathrm{ha}^{-1}$, de trigo e azevém, respectivamente. Foi utilizado o delineamento experimental de blocos casualizados, com 14 tratamentos, arranjados em um fatorial $7 \times 2$, composto pelos niveis de clodynafop-propargyl $\left(0,3,6,12,24,48\right.$ e $\left.96 \mathrm{~g} \mathrm{ha}^{-1}\right)$ isolados ou em mistura com metsulfuron-methyl $\left(2 \mathrm{~g} \mathrm{ha}^{-1}\right)$. Foi adicionado o óleo mineral Nimbus ${ }^{\circledR}$ a $0,2 \%$ para metsulfuron-methyl e $0,5 \%$ para clodinafop-propargyl e associações com outros herbicidas. Quando as plantas de trigo estavam em pleno perfilhamento e as de azevém no início deste, foram aplicados os tratamentos herbicidas. Na aplicação utilizou-se pulverizador pressurizado a $\mathrm{CO}_{2}$, munido de seis bicos tipo leque 11002, com volume de calda equivalente a $150 \mathrm{~L} \mathrm{ha}^{-1}$. A aspersão foi efetuada em ausência de vento, com temperatura média de $22{ }^{\circ} \mathrm{C}$ e $50 \%$ de umidade relativa do ar. Seguiu-se um período sem precipitações por 25 dias após aplicação dos herbicidas.

Nos estádios de alongamento de colmos e florescimento das plantas de trigo, foram utilizados o fungicida tebuconazole e o inseticida permetrina, respectivamente, para controlar ferrugem da folha (Puccinia recondida), manchas foliares (Dreschlera tritici-repentis) e lagarta-do-trigo (Pseudaletia sequax).

Efetuou-se avaliação visual de controle, aos 59 dias após aplicação dos tratamentos (DAA), da matéria verde de azevém, aos 72 DAA, e do rendimento de grãos de trigo.

Procedeu-se à análise da variância dos dados pelo teste F. No experimento em casa de vegetação, o modelo logístico de curvas de dose-resposta foi utilizado com auxílio do programa estatístico Sigma-plot, o qual estima os valores de $\mathrm{I}_{50}$ (dose necessária para reduzir ou aumentar em $50 \%$ a variável resposta). No experimento em campo, as relações entre os niveis de clodinafop-propargyl e as variáveis injúria e matéria verde foram ajustadas por meio de regressão polinomial.

\section{RESULTADOS E DISCUSSÃO}

No experimento em casa de vegetação, as aplicações de clodinafop-propargyl, isoladamente, 
em niveis superiores a $20 \mathrm{~g} \mathrm{ha}^{-1}$ resultaram em controle acima de $80 \%$ (Figura 1). No entanto, as curvas de dose-resposta em associações a metsulfuron-methyl (2 e $4 \mathrm{~g} \mathrm{ha}^{-1}$ ) e a 2,4-D demonstram que o controle de azevém de $80 \%$ ou superior somente foi obtido com a utilização de níveis de clodinafop-propargyl superiores a $20 \mathrm{~g} \mathrm{ha}^{-1}$ (Figura 1). Efeito similar é observado ao analisar as curvas de dose-resposta de matéria verde da parte aérea em relação aos niveis de clodinafop-propargyl (Figura 2), em que se constatam níveis de clodinafop para controlar azevém superiores nos tratamentos de associações com metsulfuronmethyl e 2,4-D, em relação ao uso de clodinafop-propargyl isoladamente. Na interpretação das curvas de dose-resposta para controle e matéria verde de azevém, observa-se, também, que antagonismo com o nível mais baixo de metsulfuron-methyl $\left(2 \mathrm{~g} \mathrm{ha}^{-1}\right)$ ocorre apenas quando são utilizados níveis até $24 \mathrm{~g} \mathrm{ha}^{-1} \mathrm{de}$ clodinafop-propargyl (Figuras 1 e 2). A partir do nível de $48 \mathrm{~g} \mathrm{ha}^{-1}$ também não se percebe antagonismo da associação a 2,4-D (Figuras 1 e 2). Ou seja, a elevação dos níveis do clodinafoppropargyl torna esse herbicida mais eficaz no controle de azevém e reduz a capacidade dos herbicidas latifolicidas de prejudicá-lo.



Figura 1 - Avaliação visual de controle (\%) aos 14 dias após aplicação dos herbicidas no experimento em casa de vegetação. Curso de Agronomia da UTFPR - campus Pato Branco.
Para a variável avaliação visual de controle, os $\mathrm{I}_{50}$ das associações de clodinafop-propargyl + metsulfuron-methyl $\left(2 \mathrm{~g} \mathrm{ha}^{-1} \mathrm{e} 4 \mathrm{~g} \mathrm{ha}^{-1}\right)$ e clodinafop-propargyl + 2,4-D (469 $\left.\mathrm{g} \mathrm{ha}^{-1}\right)$ foram, respectivamente, de 33,84 e $151 \%$ superiores aos de clodinafop-propargyl aplicado isoladamente (Tabela 1). Já para a variável matéria verde de azevém, os $\mathrm{I}_{50}$ das misturas supracitadas foram, respectivamente, 119, 244 e $72 \%$ superiores aos de clodinafop-propargyl aplicado isoladamen te.

A análise das Figuras 1 e 2 demonstra que o estabelecimento de niveis de clodinafoppropargyl e de suas associações para controlar $80 \%\left(\mathrm{I}_{80}\right)$ das plantas de azevém - valores mínimos aceitáveis em termos práticos - demandaria valores superiores aos de $\mathrm{I}_{50}$. Como $80 \%$ representa um local de maior afastamento entre as curvas logísticas, em comparação a 50\% (Figuras 1 e 2), isso implica ampliação das diferenças entre os niveis de clodinafop-propargyl associados e clodinafop-propargyl isolado, necessária para atingir o nível de controle mais elevado.

No experimento em campo, as aplicações de clodinafop-propargyl, isoladamente, em doses entre 40 e $50 \mathrm{~g} \mathrm{ha}^{-1}$, faixa recomendada



Figura 2 -Matéria verde da parte aérea $\left(\mathrm{g}\right.$ planta $\left.^{-1}\right)$ de azevém aos 28 dias após aplicação dos herbicidas. Curso de Agronomia da UTFPR - campus Pato Branco. 
Tabela 1 - Níveis de clodynafop-propargyl necessários para reduzir em 50\% o desenvolvimento de azevém, quando este herbicida é aplicado isoladamente ou em associações com metsulfuron-methyl ou 2,4-D. Curso de Agronomia da UTFPR - campus Pato Branco

\begin{tabular}{|l|c|c|c|c|}
\hline \multicolumn{1}{|c|}{ Tratamento } & $\begin{array}{c}\mathrm{I}_{50}{ }^{*} \text { para injúria aos } \\
\text { 14 DAA } \\
\left(\mathrm{g} \mathrm{ha}^{-1}\right)\end{array}$ & $\begin{array}{c}\text { \% em relação a } \\
\text { clodinafop } \\
\text { isolado }\end{array}$ & $\begin{array}{c}\mathrm{I}_{50}{ }^{*} \text { para matéria } \\
\text { verde } \\
\left(\mathrm{g} \mathrm{ha}^{-1}\right)\end{array}$ & $\begin{array}{c}\text { \% em relação ao } \\
\text { clodinafop } \\
\text { isolado }\end{array}$ \\
\hline Clodinafop & 5,5 & 100 & 3,2 & 100 \\
\hline Clodinafop + metsulfuron $2 \mathrm{~g} \mathrm{ha}^{-1}$ & 7,3 & 133 & 7,0 & 219 \\
\hline Clodinafop + metsulfuron 4 $\mathrm{g} \mathrm{ha}^{-1}$ & 10,1 & 184 & 11,0 & 344 \\
\hline Clodinafop-propargyl + 2,4-D & 13,8 & 251 & 5,5 & 172 \\
\hline
\end{tabular}

* Dose para produzir $50 \%$ de injúria ou reduzir $50 \%$ da matéria verde de azevém.

pelo fabricante para controle de azevém, resultaram em controle visual de apenas 35 a $42 \%$ (Figura 3), em contraste com os niveis de controle obtidos em casa de vegetação. Essas diferenças de eficiência justificam-se, em parte, porque em campo, logo após a aplicação dos herbicidas, ocorreu período de ausência de chuvas de cerca de 25 dias, o que, provavelmente, reduziu as taxas metabólicas das plantas de azevém, ocasionando redução nos niveis de controle. Também, em campo, parte do clodinafop-propargyl pode ser interceptada pelas plantas de trigo, reduzindo sua eficácia em atingir as plantas de azevém.

As associações de clodinafop-propargyl (40 e $50 \mathrm{~g} \mathrm{ha}^{-1}$ ) com metsulfuron-methyl reduziram os niveis de controle de azevém para, respectivamente, 23 e $27 \%$. Isso corresponde a uma redução da eficiência de aproximadamente

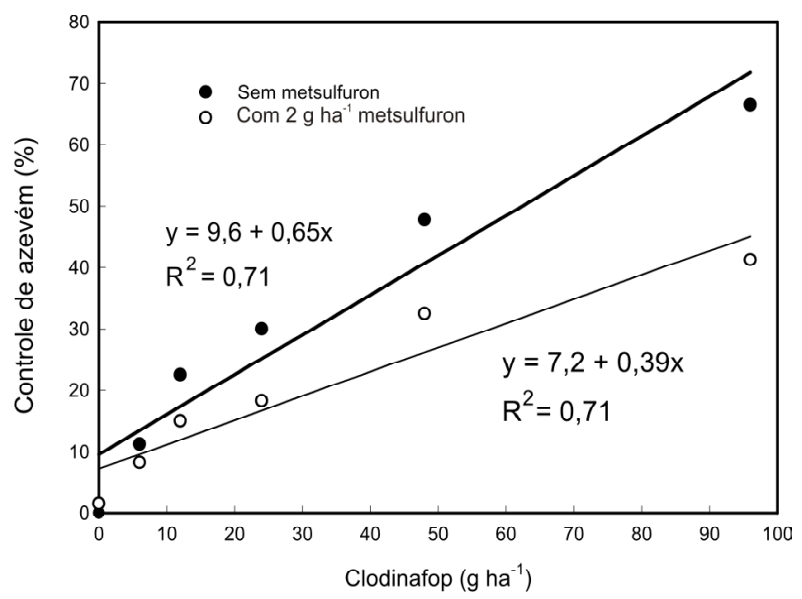

Figura 3 - Avaliação de controle (\%) das plantas de azevém aos 59 dias após aplicação isolada de clodinafop-propargyl ou em associação com metsulfuron-methyl $\left(2 \mathrm{~g} \mathrm{ha}^{-1}\right)$. Curso de Agronomia da UTFPR - campus Pato Branco.
$35 \%$ em relação às mesmas doses utilizadas em aplicações isoladas de clodinafop-propargyl. No experimento em campo, constatou-se pequena atrofia das plantas de azevém, sem que houvesse a morte destas.

Os resultados de controle das plantas de azevém estão de acordo com dados de avaliação da matéria verde das plantas (Figura 4). Ocorreu redução da matéria verde de azevém em função da elevação dos niveis de clodinafop-propargyl - relação ajustada pelo modelo quadrático. No entanto, não houve variação da matéria verde das plantas de azevém com a elevação dos níveis de clodinafop-propargyl quando em associação com metsulfuron-methyl (Figura 4), o que evidencia o antagonismo.

A descrição de antagonismo entre herbicidas graminicidas e latifolicidas, em várias

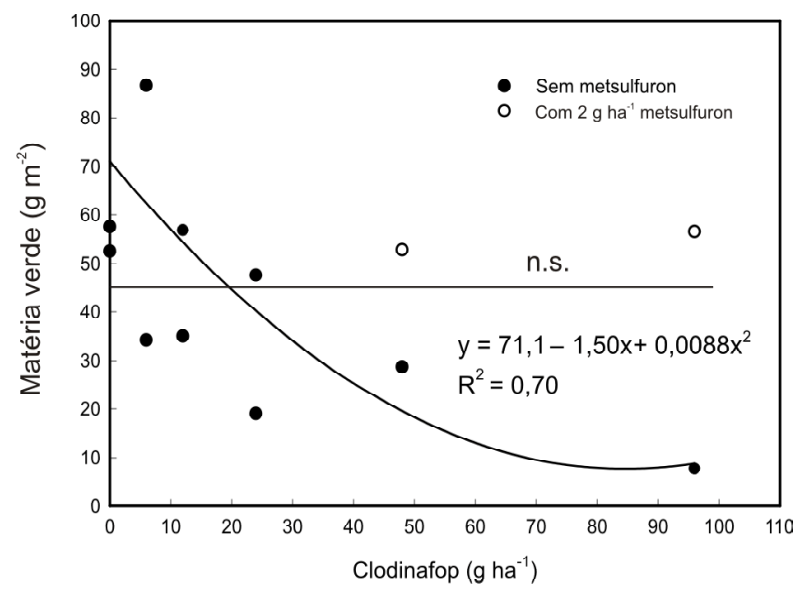

Figura 4 - Matéria verde das plantas de azevém aos 72 dias após aplicação isolada de clodinafop-propargyl ou em associação com metsulfuron-methyl $\left(2 \mathrm{~g} \mathrm{ha}^{-1}\right)$. Curso de Agronomia da UTFPR - campus Pato Branco. 
espécies cultivadas, é observada com freqüência na literatura (Olson \& Nalejawa, 1981; Deschamps et al., 1990; Holshouser \& Coble, 1990; Vidrine et al., 1995; Burke et al., 2004; Scherder et al., 2005). Nos Estados Unidos, Minton et al. (1989) demonstraram que a associação de clodinafop-propargyl com herbicidas inibidores da ALS comprometeu o controle de Echinochloa crus-galli em taxas que variaram de 5 a $45 \%$. Na região sudoeste do Paraná, a associação de clodinafop-propargyl $\left(36 \mathrm{~g} \mathrm{ha}^{-1}\right)$ com metsulfuron-methyl $\left(4 \mathrm{~g} \mathrm{ha}^{-1}\right)$ reduziu o nível de controle de azevém em $28 \%$, em relação à testemunha com clodinafop isolado (Mattei et al., 2004).

Em casos de antagonismo entre clodinafoppropargyl e latifolicidas, pelo menos duas estratégias poderiam ser adotadas. A primeira delas seria a elevação das doses de clodinafoppropargyl, quando em associação, para produzir o mesmo nivel de controle de azevém obtido com sua aplicação isolada. A segunda alternativa seria respeitar um intervalo minimo entre as aplicações de herbicidas desses mecanismos de ação, o que implicaria custos adicionais com a operação extra de aplicação e os danos decorrentes do amassamento das plantas de trigo. Mattei et al. (2004) demonstraram que intervalos de 12 dias antes ou após a aplicação de metsulfuron-methyl foram suficientes para evitar a redução na eficiência de clodinafoppropargyl, possibilitando, dessa forma, controle de azevém acima de $80 \%$. Como no trabalho de Mattei et al. (2004) não foram testados intervalos menores, não está afastada a hipótese de que eles sejam suficientes para evitar os problemas de antagonismo.

A recomendação ou não de associação entre esses herbicidas necessita de análise econômica que considere, de um lado, o valor despendido com a elevação da dose de clodinafop-propargyl para superar o antagonismo e, de outro, os custos adicionais com as operações de aspersão dos herbicidas e amassamento. Considerando os valores de $\mathrm{I}_{50}$ de injúria das associações em relação à aplicação isolada em casa de vegetação (Tabela 1), haveria necessidade de elevação em 33, 84 e 151\% da dose de clodinafop-propargyl, respectivamente para os dois niveis de metsulfuronmethyl (2 e $4 \mathrm{~g} \mathrm{ha}^{-1}$ ) e 2,4-D, para compensar o antagonismo. Considerando-se $45 \mathrm{~g} \mathrm{ha}^{-1} \mathrm{a}$ dose recomendada pelo fabricante para controlar azevém a campo e o valor de clodinafoppropargyl (U\$ 160,00/240 g), o custo por hectare corresponderia a U\$30. As elevações nas doses corresponderiam a valores de U\$ 9,90, 25,20 e 45,30. Por outro lado, o custo médio para pulverizar um hectare de lavoura na região de Pato Branco (PR) é de aproxi madamente U\$ 10,00. Portanto, considerando-se que uma segunda operação para aplicação de herbicida latifolicida resulte em acréscimo pouco significativo nos danos por amassamento de trigo, os resultados de casa de vegetação indicam que a decisão de elevar a dose de clodinafoppropargyl ou executar uma segunda operação de pulverização dependeria da dose de latifolicida empregado em associação ao clodinafoppropargyl, caso esse seja metsulfuron-methyl. No entanto, o cálculo anterior considera os valores de $I_{50}$ para as associações, tendo como referência a aplicação isolada de clodinafoppropargyl. Em caso de utilização de $\mathrm{I}_{80}$ como parâmetro referencial de controle, is so implicaria ampliação das diferenças entre os niveis de clodinafop-propargyl associados e clodinafop-propargyl isolado, ou seja, a elevação das doses de clodinafop-propargyl em associação a latifolicidas deveria ser ampliada para superar o antagonismo, o que inviabilizaria essa estratégia de ação pelo custo adicional gerado.

Além disso, o experimento em campo demonstra que mesmo o nivel mais baixo de metsulfuron-methyl ( $\left.2 \mathrm{~g} \mathrm{ha}^{-1}\right)$ foi capaz de prejudicar a ação do graminicida (Figuras 3 e 4). Considerando os valores de $\mathrm{I}_{50}$ para matéria verde (Tabela 1), que representam acréscimos nas doses de clodinafop-propargyl superiores aos considerados nos cálculos anteriores para superar o antagonismo, e os resultados obtidos em campo, constata-se que, para qualquer dos herbicidas e doses testados, seria mais compensador executar uma segunda operação para controlar plantas daninhas latifoliadas.

No presente trabalho, em campo, a competitividade exercida pelas plantas de azevém das parcelas sem controle e daquelas com controle deficiente não prejudicou expressivamente o rendimento de grãos de trigo (Tabela 2). O principal fator que explica a baixa interferência do azevém com trigo, no presente experimento, foram características competitivas das plantas 
Tabela 2 - Rendimento de grãos de trigo, cultivar CD-205, em função de diferentes níveis de clodinafop-propargyl e metsulfuronmethyl. Curso de Agronomia da UTFPR - campus Pato Branco

\begin{tabular}{|c|c|c|}
\hline \multirow{2}{*}{ Tratamento } & Sem metsulfuron & Com metsulfuron \\
\hline & \multicolumn{2}{|c|}{ Rendimento de grãos $\left(\mathrm{kg} \mathrm{ha}^{-1}\right)$} \\
\hline Testemunha sem controle & $3.067^{\text {n.s. }}$ & $3.484^{\text {n.s. }}$ \\
\hline Clodinafop $6 \mathrm{~g} \mathrm{ha}^{-1}$ & 3.502 & 3.597 \\
\hline Clodinafop $12 \mathrm{~g} \mathrm{ha}^{-1}$ & 3.477 & 3.298 \\
\hline Clodinafop $24 \mathrm{~g} \mathrm{ha}^{-1}$ & 3.385 & 3.767 \\
\hline Clodinafop $48 \mathrm{~g} \mathrm{ha}^{-1}$ & 3.157 & 3.032 \\
\hline Clodinafop $96 \mathrm{~g} \mathrm{ha}^{-1}$ & 3.290 & 3.235 \\
\hline
\end{tabular}

n.s. Não-significativo pelo teste F.

de trigo, especialmente o desenvolvimento inicial mais rápido em relação ao azevém (dados não quantificados). Dentre os fatores que influenciam a capacidade competitiva de azevém com trigo estão as condições de ambiente, o cultivar utilizado e a densidade das plantas competidoras. Densidades de azevém anual de 29 a 118 plantas $\mathrm{m}^{-2}$ reduziram o rendimento de trigo entre 7 e 50\% (Appleby et al., 1976). A cada 10 plantas $\mathrm{m}^{-2}$ de azevém, Liebel \& Worsham (1987) constataram redução na produtividade de trigo de 4,2\%. No entanto, Fleck (1980) demonstrou que cultivares de trigo mais competitivos podem não apresentar redução do rendimento de grãos pela competição com niveis crescentes de plantas de azevém.

Considerando apenas o rendimento de grãos de trigo, o fato de o controle deficiente não resultar em sua redução poderia induzir à decisão de não elevar as doses ou aplicar os herbicidas em duas operações, ou mesmo não adotar medidas de controle. No entanto, a presença de plantas de azevém dificultaria a produção de sementes de trigo e permitiria a reinfestação de azevém em safras futuras, o que, provavelmente, justificaria a aplicação dos herbicidas em duas operações distintas para manter elevados os niveis de controle dessa espécie.

\section{LITERATURA CITADA}

APPLEBY, A. P. et al. Winter wheat yield reduction from interference by Italian ryegrass. Agron. J., v. 68, p. 463-466, 1976.

BAILEY, W. A.; WILSON, H. P. Control of italian ryegrass (Lolium multiflorum) in wheat (triticum aestivum) with postemergence herbicides. Weed Technol., v. 17, p. 534542, 2003.
BRASIL. Ministério da Agricultura, Pecuária e Abastecimento. Instrução Normativa n. 25. Padrão para produção e comercialização de sementes de trigo e trigo duro. Diário Oficial da República Federativa do Brasil, Brasília, DF 20 dez. 2005.

BROMMER, C. L. et al. Antagonism of clefoxydim by selected broadleaf herbicides and the role of ethanol. Weed Sci., v. 48, p. 181-187, 2000.

BURKE, I. C. et al. CGA-362622 antagonizes annual grass control with clethodim. Weed Technol., v. 16, p. 749-754, 2002.

COLBY, S. R. Calculating synergistic and antagonistic responses of herbicide combinations. Weeds, v. 15, n. 1, p. 20-22, 1967.

CULPEPPER, A. S. et al. Influence of bromoxynil on annual grass control by graminicides. Weed Sci., v. 47, p. 123-128, 1999.

CULPEPPER, A.S. et al. Interactions of bromoxynil and postemergence graminicides in large crabgrass (Digitaria sanguinalis). Weed Technol., v. 12, p. 554-559, 1998

DESCHAMPS, R. J. A. et al. Antagonistic effect of MCPA on fenoxaprop activity. Weed Sci., v. 38, p. 62-66, 1990.

FLECK, N. G. Competição de azevém (Lolium multiflorum L.) com duas cultivares de trigo. Planta Daninha, v. 3, p. 61-67, 1980.

HOLM, F. et al. Defining optimum herbicide rates and timing for wild oat (Avena fatua) control in spring wheat (Triticum aestivum). Weed Technol., v. 14, p. 167-175, 2000.

HOLSHOUSER, D. L.; COBLE, H. D. Compatibility of sethoxydim with five postemergence broadleaf herbicides. Weed Technol., v. 4, p. 128-133, 1990.

HOSKINS, A. J. et al. Control of Italian ryegrass (Lolium multiflorum) in winter wheat. Weed Technol., v. 19, p. 261265, 2005. 
KRUSE, N. D.; VIDAL, R. A.; TREZZI, M. M. Curvas de resposta e isobolograma como forma de descrever a associação de herbicidas inibidores do fotossistema II e da

síntese de carotenóides. Planta Daninha, v. 24, p.579-587, 2006.

KUNZ, R. P. et al. Interação entre o herbicida clodinafop propargil e os latifolicidas metsulfuron e 2,4-D no controle de Avena sativa e Avena strigosa, na cultura do trigo. In: CONGRESSO BRASILEIRO DA CIÊNCIA DAS

PLANTAS DANINHAS, 24., 2004, Águas de São Pedro.

Resumos... Viçosa, MG: Sociedade Brasileira da Ciência das Plantas Daninhas, 2004. p. 260.

LIEBEL, R.; WORSHAM, A. D. Effect of chlorsulfuron on diclofop phytotoxicity to Italian ryegrass (Lolium multiflorum). Weed Sci., v. 35, p. 383-387, 1987.

LIMPEL, L. E. et al. Weed control by dimethyl tetrachloroterephthalate alone and in certain combinations. Proc. North. Weed Control Conference, v. 16, p. 48-53, 1962.

MATTEI, D. et al. Eficiência no controle de azevém (Lolium multiflorum) e seletividade para o trigo (Triticum aestivum) da associação dos herbicidas clodina fop-propargyl e metsulfuron-methyl. In: CONGRESSO BRASILEIRO DA CIÊNCIA DAS PLANTAS DANINHAS, 24., 2004, Águas de São Pedro. Resumos... Viçosa, MG: Sociedade Brasileira da Ciência das Plantas Daninhas, 2004. p. 138.
MINTON, B. W. et al. Barnyard grass (Echinochloa crusgalli) control with grass and broadleaf weed herbicide combinations. Weed Sci., v. 37, p. 223-227, 1989.

NUÑES, J. O.; SANCHEZ, J. D. Clodinafop, nuevo herbicida para controlar selectivamente malezas gramíneas en trigo. II. Tolerancia de cultivares primaverales y alternativos. Agric. Técnica, v. 58, 1998. Ministério da Agricultura do Chile. 1998. Disponível em: <http://www.inia.cl/at/ espanol>. Acesso em: 29/4/2006.

OLSON, W. A.; NALEJAWA, J. D. Antagonistic effects on MCPA on wild oat (Avena fatua) control with diclofop.

Weed Sci., v. 29, p. 566-571, 1981.

SCHERDER, E. F. et al. Antagonism of cyhalofop grass activity by halosulfuron, triclopyr and propanil. Weed Technol., v. 19, p. 934-941, 2005.

VENCILL, W. K. Herbicide Handbook. 8.ed. Lawrence: Western Social Science Association, 2002. p. 493.

VIDAL, R. A; MEROTTO JR., A. Inibidores da ALS. In: VIDAL, R. A.; MEROTTO JR., A. Herbicidologia. Porto Alegre: Edição dos Autores, 2001. p. 15-24.

VIDRINE, P. R. et al. Grass control in soybean (Glycine max) with graminicides applied alone and in mixtures. Weed Technol., v. 9, p. 68-72, 1995. 